\title{
INFLUENCIA DE LA GEOMETRÍA EN LA RESISTENCIA A LA FLEXIÓN DE LOS ENGRANAJES PLÁSTICOS
}

\author{
INFLUENCE OF GEOMETRY IN THE RESISTANCE OF \\ PLASTICS GEARS
}

\author{
Jorge Laureano Moya Rodríguez ${ }^{1}$, Tito Roberto Vílchez Vílchez ${ }^{2}$ \\ RESUMEN
}

En el presente trabajo se analiza el tema relacionado con las modificaciones geométricas del perfil del diente de engranajes plásticos cilíndricos de dientes rectos mediante el método de los elementos finitos y su influencia en la resistencia a la flexión. El objetivo fundamental del estudio es, desarrollar un procedimiento para determinar la influencia de la asimetría y la corrección del dentado en la resistencia a la flexión de los dientes de los engranajes plásticos. En esta investigación se ha desarrollado el cálculo de los esfuerzos de flexión, mediante procedimientos matemáticos y el uso del software con el que se ha logrado incrementar la resistencia a la flexión debido a la corrección del dentado (para un factor de corrección de 0 a 0,7) y asimismo la determinación del factor de forma en los engranajes cilíndricos de dientes rectos asimétricos que constituye una innovación científica. Los resultados alcanzados satisfacen a los objetivos generales y específicos del estudio, con el cual se demuestra que las innovaciones contribuirán a una mejorar el desarrollo tecnológico de la industria y del país.

Palabras clave.- Engranajes, Polímeros, Simulación numérica, Cálculo analítico, Método experimental, $M E F$.

\begin{abstract}
In this paper we can examine the influence of geometric changes in the straight tooth profiles of cylindrical plastic gears on its bending resistance by the finite element method and its influence on the resistance to bending. The main objective of the study is to develop a procedure to determine the influence of asymmetry and the teeth modification with regard to the flexural strength of the plastic gear teeth. In this study we have calculated the tooth bending stresses using mathematical procedures and use of software that has allowed to increase the flexural strength due to the teeth modification (by a modification factor from 0 to 0,7) and also determine the shape factor for the cylindrical asymmetrical straight teeth gears, and which constitutes a scientific innovation. The results obtained satisfy the general and specific objectives of the study, which demonstrates that innovations will contribute to the industry and the country technological development.
\end{abstract}

Keywords.- Gears, Polymers, Numerical simulation, Analytical calculation, Experimental method, MEF.

\section{INTRODUCCIÓN}

La búsqueda constante, y el mejoramiento de las producciones mecánicas forman parte del andar diario en el campo de la Ingeniería Mecánica. Los engranajes como parte indispensable de los mecanismos y las máquinas, son en el mundo objeto de importantes investigaciones en aras de lograr mejores diseños, nuevos materiales, mayor eficiencia, etc.

${ }^{1}$ Dr. Vice Decano de Investigaciones y Posgrado Facultad de Mecánica Universidad Central Marta Abreu de las Villas Cuba, ${ }^{2}$ M.Sc Ing. Docente investigador de la Facultad de Ingeniería Mecánica de la Universidad Nacional de Ingeniería. 
El mejoramiento de las producciones y el aumento de la eficiencia, son además una necesidad impostergable para aumentar la cantidad de fondos exportables de cualquier país. Desarrollar una política efectiva de sustitución de importaciones, elevar la eficiencia de la producción social y mejorar el nivel de vida de la población son objetivos primordiales hoy en día.

En la fabricación de partes y sistemas de partes de todo tipo para los más variados usos e intereses, es usado el engranaje tanto a escala nacional como internacional con lo cual se justifican las investigaciones que en este tema se realicen.

El estudio de los diferentes tipos de engranajes, variaciones de los perfiles, así como la situación del plástico en sus más disimiles variantes ha cobrado fuerza en este último milenio. El logro de perfiles que dan paso a un espectro más amplio de utilización de los polímeros es realmente un paso impresionante de los científicos en la segunda mitad del siglo pasado y a comienzos de este.

Es importante destacar que el plástico ha marcado una era, por lo cual, el empleo de este material no se puede desvincular de ninguna manera de esferas tan importantes como la producción de bienes de consumo, equipos electrodomésticos, industria automovilística y en la industria alimenticia por solo citar algunas. Es por ello que la comunidad científica a nivel mundial en cada una de sus ramas se proyecta para el futuro, teniendo en cuenta las materias primas plásticas atendiendo a su bajo costo de producción en comparación como lo sería con el acero, el aluminio u otro material convencional.

\section{TIPOS DE ENGRANAJES}

\section{Engranajes simétricos}

Tradicionalmente los engranajes se han fabricado simétricamente. En estos engranajes, los ángulos de presión son iguales para ambos lados del diente $\mathrm{y}$ por ende, las superficies de estos lados son simétricas.

En la literatura existen múltiples trabajos donde se evalúa y perfecciona el cálculo de resistencia a la flexión de engranajes metálicos simétricos [1], inclusive, se han realizado tesis doctorales, cuyo contenido esencial ha sido la creación de modelos más precisos para el cálculo a presión de los engranajes cilíndricos de dientes rectos metálicos[2, 3 y 4].

Una forma de incrementar la capacidad de carga es modificar la geometría de la involuta. Esta ha sido una práctica normal en el diseño de los engranajes sofisticados por muchos años. La nomenclatura para describir este tipo de modificaciones, es bastante confusa en lo que se refiere a las modificaciones de addendum, corrección del dentado, etc.

\section{Engranajes asimétricos}

Una modificación adicional que puede incrementar notablemente la capacidad de transmisión de potencia es hacer los engranajes con dientes asimétricos, es decir, con diferentes ángulos del perfil para cada lado del diente [13 y 15]. Se muestra un ejemplo en la Figura 1.

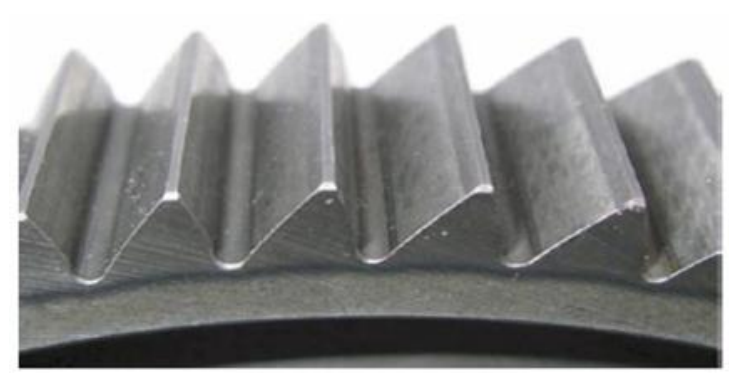

Fig. 1 Dientes asimétricos.

Con dos ángulos diferentes, uno de ellos puede ser elegido para cumplir con los objetivos del diseño para la transmisión de potencia y el otro para compensar las desventajas del primero.

El grado de asimetría y selección del perfil conductor depende de la aplicación del engranaje. El esfuerzo de flexión es importante, entonces el perfil con ángulo de presión bajo, es preferible para el lado conductor, a este tipo de dientes también se les conoce como "reforzados" o "apuntalados" [1 y 16], en ellos se considera generalmente, un perfil con ángulo de presión de $20^{\circ}$ para el lado conductor y un perfil con ángulo de presión alto para el otro lado de los dientes. De esta forma disminuyen los esfuerzos por flexión y se mantienen los esfuerzos de contacto en el mismo nivel que para dientes simétricos con el mismo ángulo de presión. 
Consideraciones en la corrección del dentado.- El perfil de evolvente para los dientes de los engranajes es actualmente muy utilizado por las ventajas que ofrece en cuanto a rendimiento, resistencia y duración, dado que con este perfil se tienen bajas velocidades de deslizamiento y suficientes radios de curvatura en los puntos de contacto. Adicionalmente, el engranaje de perfil de evolvente permite la corrección o mejoramiento del perfil de los dientes, es decir, el empleo de las partes de la evolvente que permiten incrementar significativamente la capacidad de transmisión de carga o potencia, debido a que el diente se hace más grueso cerca de su base, se puede reducir el número de dientes y aumentar respectivamente el modulo y se aumentan los radios de curvatura de las superficies de evolvente. [7, 8 y 9].

Cuando mayor es el desplazamiento de la herramienta durante el tallado, el perfil de los dientes se va trazando por la parte de la evolvente más distante de la circunferencia principal. En este caso, los radios de curvatura de los dientes aumentan, el diente se hace más grueso cerca de la base y más delgado en el vértice. La corrección de los engranajes puede ser de altura o angular. En el caso de la corrección de la altura, la rueda se talla con desplazamiento negativo de la herramientacremallera y el piñón con desplazamiento positivo de la misma magnitud. Con esta corrección, el espesor de los dientes por la circunferencia primitiva del piñón aumenta, mientras que el espesor de los dientes de la rueda disminuye, pero la suma de estos espesores por la misma circunferencia de los dientes a engranar queda constante e igual al paso, por lo tanto, no es necesario separar los ejes de las ruedas, las circunferencias de base coinciden con las primitivas, tanto en las ruedas corregidas como en las no corregidas. El ángulo de engrane no varía. Ha sido demostrado a través de los años la influencia positiva que tienen las correcciones en el mejoramiento de las transmisiones por engranajes, como se muestra en la Figura 2 [10].

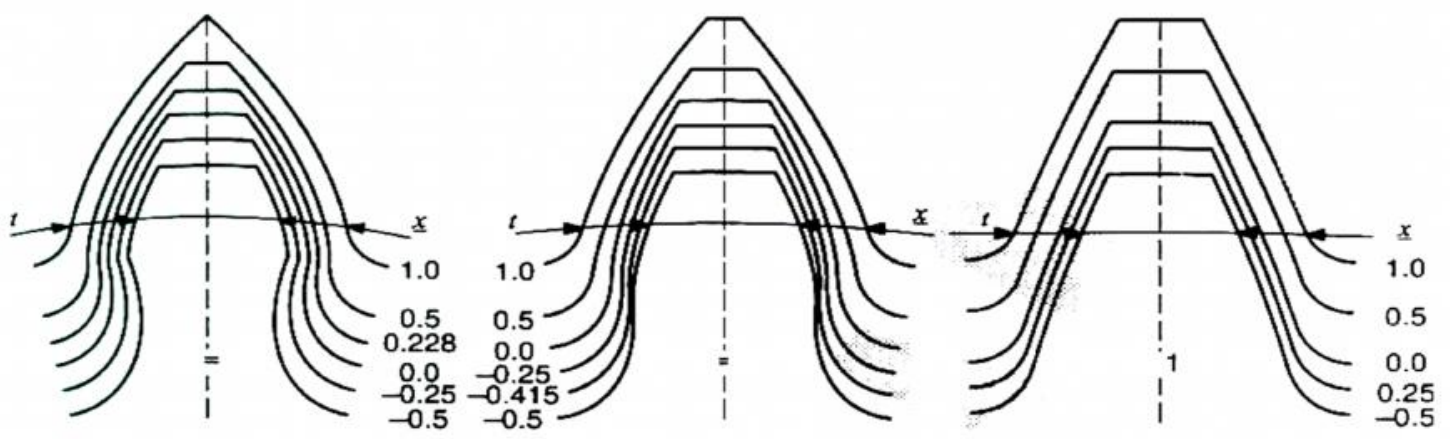

Fig. 2 Modificación del perfil del diente de acuerdo a la corrección.

Determinación del factor de Lewis o factor de Forma en engranajes asimétricos.- Se utilizó el Software StatGraphics Plus 4.1, hallándose las ecuaciones de regresión para obtener los valores del factor de Lewis (Y) para dientes asimétricos en función del número de dientes y el coeficiente de asimetría. Para ser más preciso, referente al valor de este factor, se establecieron tres expresiones de acuerdo al rango de número de dientes:

$10 \leq z \leq 35,40 \leq z \leq 70$ y $75 \leq z \leq 100$.

Para $10 \leq \mathrm{Z} \leq 35$ (Con un 98,9\% de Confianza y un error absoluto de 0,0053 )

$Y=-0,315949+0,0269414 . Z+0,405287 . C-0,000462649 . Z^{2}-0,103118 . C^{2}$

Para $40 \leq Z \leq 70$ (Con un $99.13 \%$ de Confianza y un error absoluto de 0,00058 )

$Y=-0,0543935+0,011263 . Z+0,0685655 . C-0,0000845454 . Z^{2}+0,0404041 . C^{2}$

Para $75 \leq \mathrm{Z} \leq 100$ (Con un $99.13 \%$ de Confianza y un error absoluto de 0,00058 ) 
$Y=-0,0599137+0,00834091 \cdot Z+0,028519 . C-0,0000413636 . Z^{2}+0,0581197 . C^{2}$

De los cálculos obtenidos se construye la Tabla 1.

Tabla 1. Valores del factor de forma para dientes asimétricos.

\begin{tabular}{lllllllllllllll}
\hline \multirow{2}{*}{$\mathrm{C}$} & \multicolumn{1}{c}{ Valores del factor de Forma de acuerdo al número de dientes $\mathrm{Z}$ y coeficiente de asimetría (C) } \\
& $\mathrm{Z}=10$ & $\mathrm{Z}=12$ & $\mathrm{Z}=15$ & $\mathrm{Z}=20$ & $\mathrm{Z}=30$ & $\mathrm{Z}=40$ & $\mathrm{Z}=50$ & $\mathrm{Z}=60$ & $\mathrm{Z}=70$ & $\mathrm{Z}=80$ & $\mathrm{Z}=90$ & $\mathrm{Z}=100$ \\
\hline 1 & 0.209 & 0.243 & 0.286 & 0.340 & 0.378 & 0.370 & 0.406 & 0.426 & 0.429 & 0.429 & 0.442 & 0.447 \\
1.05 & 0.219 & 0.253 & 0.296 & 0.350 & 0.388 & 0.377 & 0.414 & 0.434 & 0.436 & 0.437 & 0.450 & 0.455 \\
1.1 & 0.228 & 0.262 & 0.305 & 0.359 & 0.397 & 0.385 & 0.422 & 0.441 & 0.444 & 0.444 & 0.457 & 0.462 \\
1.15 & 0.237 & 0.270 & 0.314 & 0.368 & 0.406 & 0.393 & 0.430 & 0.449 & 0.452 & 0.452 & 0.465 & 0.470 \\
1.2 & 0.245 & 0.279 & 0.322 & 0.376 & 0.414 & 0.401 & 0.438 & 0.457 & 0.460 & 0.461 & 0.474 & 0.478 \\
1.25 & 0.253 & 0.286 & 0.330 & 0.383 & 0.421 & 0.410 & 0.446 & 0.466 & 0.469 & 0.469 & 0.482 & 0.487 \\
1.3 & 0.260 & 0.293 & 0.337 & 0.390 & 0.429 & 0.418 & 0.455 & 0.474 & 0.477 & 0.478 & 0.491 & 0.496 \\
1.35 & 0.266 & 0.300 & 0.343 & 0.397 & 0.435 & 0.427 & 0.464 & 0.483 & 0.486 & 0.487 & 0.500 & 0.505 \\
1.4 & 0.272 & 0.306 & 0.349 & 0.403 & 0.441 & 0.436 & 0.473 & 0.492 & 0.495 & 0.496 & 0.510 & 0.514 \\
1.45 & 0.278 & 0.312 & 0.355 & 0.409 & 0.447 & 0.445 & 0.482 & 0.501 & 0.504 & 0.506 & 0.519 & 0.524 \\
1.5 & 0.283 & 0.317 & 0.360 & 0.414 & 0.452 & 0.455 & 0.491 & 0.511 & 0.514 & 0.516 & 0.529 & 0.534 \\
\hline
\end{tabular}

En la Figura 3, se muestra cómo varía el factor de coeficientes de asimetría.

Forma, para diferentes números de dientes y

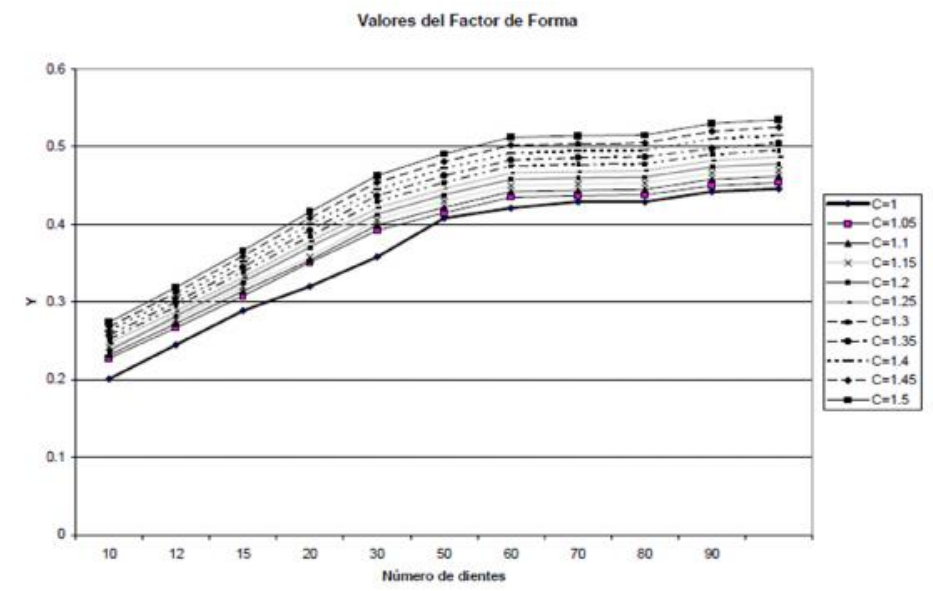

Fig. 3 Variación del factor de forma de acuerdo al número de dientes y el coeficiente de asimetría.

Otra vía para poder hallar los valores del factor de Lewis para dientes asimétricos es construyendo a escala los mismos.

Para comparar los resultados, haciendo representaciones graficas, se necesita

construir geométricamente las diferentes asimetrías de los dientes y tener en cuenta los números de dientes que puede alcanzar las ruedas según se muestra en la Figura 4.

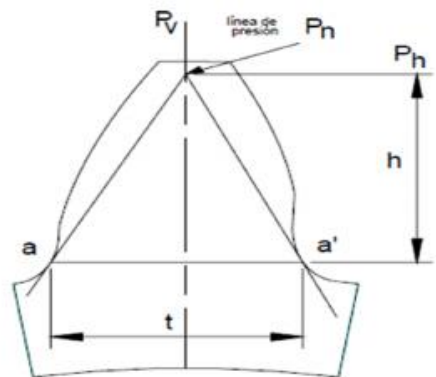

Fig. 4 Determinación del factor de Lewis para engranajes asimétricos. 
A manera de ejemplo para un coeficiente de asimetría $C=1.35\left(\alpha \mathrm{cmen}=20^{\circ}\right.$ y $\left.\alpha \mathrm{cmay}=27^{\circ}\right)$ y números de dientes de $Z=30, Z=40, Z=50, Z=60$ y $\mathrm{Z}=90$, se determinó el valor de $Y_{\mathrm{F}}$ gráficamente por la expresión:

$$
Y_{F}=\frac{t^{2}}{6 h}
$$

Hay que destacar que este valor (para módulo y ancho unitarios), como es lógico, será algo menor que el valor del factor de Lewis $Y_{F}$ calculado, ya que no tiene en cuenta el efecto de la disminución de la tensión en el pie del diente debido a la componente vertical de la fuerza y a la excentricidad.

En la Tabla 2 se muestra una comparación entre los valores del factor de forma calculados por la expresión (1) con el algoritmo y programa desarrollado $\left(\mathrm{Y}_{\mathrm{Fr}}\right)$, el establecido por las ecuaciones de regresión obtenidas del StatGraphics (Ysg) y el efectuado por los dibujos realizados $\left(\mathrm{Y}_{\mathrm{Fd}}\right)$.

Tabla 2. Valores del coeficiente de Lewis en dientes asimétricos por diferentes métodos.

\begin{tabular}{|c|c|c|c|c|c|c|c|c|c|c|c|c|}
\hline & \multicolumn{3}{|c|}{$\mathrm{Z}=30$} & \multicolumn{3}{|c|}{$Z=40$} & \multicolumn{3}{|l|}{$Z=50$} & \multicolumn{3}{|c|}{$Z=90$} \\
\hline Y & & $n$ & $n$ & & $n$ & & & $n$ & $\bullet$ & & $\curvearrowleft$ & \\
\hline & 一 & $\stackrel{-}{-}$ & $\because$ & - & $?$ & $\because$ & - & $\stackrel{?}{-}$ & - & - & 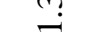 & ? \\
\hline & 11 & II & II & II & $\pi$ & II & II & ॥ & II & II & II & ॥ \\
\hline $\mathrm{Y}_{\mathrm{Fr}}$ & 0,378 & 0,435 & 0.452 & 0,37 & 0.427 & 0.455 & $\underset{0.406}{\cup}$ & $\underset{464}{\cup}$ & $\underset{0.491}{\cup}$ & $\underset{0.442}{\cup}$ & $\stackrel{u}{50}$ & $\underset{520}{U}$ \\
\hline $\mathrm{y}_{\mathrm{sg}}$ & 0,378 & 0,435 & 0,451 & 0,37 & 0,427 & 0,454 & 0,406 & 0,463 & 0,491 & 0,445 & 0,503 & 0,532 \\
\hline $\mathrm{y}_{\mathrm{Fd}}$ & - & 0,403 & - & - & 0,413 & - & - & 0,428 & - & - & 0,468 & - \\
\hline
\end{tabular}

Evaluación de la asimetría y la corrección en la resistencia a la flexión de los engranajes plásticos mediante el método de elementos finitos.- Para evaluar la influencia de la corrección, la asimetría del diente y la combinación de ambas en la resistencia a la flexión de los engranajes plásticos se realizó un experimento usando el método de los elementos finitos MEF. Se eligió un valor de módulo, de número de dientes, de ancho de cara y de potencia a trasmitir y se analizó como variaban las tensiones en el pie del diente para diferentes valores de corrección y de asimetría del perfil.

Para el experimento el ángulo de ataque del perfil se varió desde 17 grados hasta 27 grados, y el ángulo de respaldo en ese mismo rango [5].
Para cada combinación de ángulo de ataque con ángulo de respaldo se evaluó el comportamiento de la corrección, introduciendo coeficientes o factores de corrección que variaron de $\mathrm{X}=0$ hasta $\mathrm{X}=0,8$.

Se utilizó una Potencia de $100 \mathrm{~kW}$ a una velocidad de giro del piñón de 720 RPM, con módulo 4mm y 17 dientes, valores típicos de una transmisión por engranajes plásticos de transmisión de potencia.

En la Figura 5, se muestra como varían las Tensiones normales $\sigma_{\mathrm{z}}$ en el eje $\mathrm{z}$ a lo largo del diente para una rueda de módulo $4 \mathrm{~mm}$ y 17 dientes, con un ángulo del perfil de 20 grados y sin corrección. 


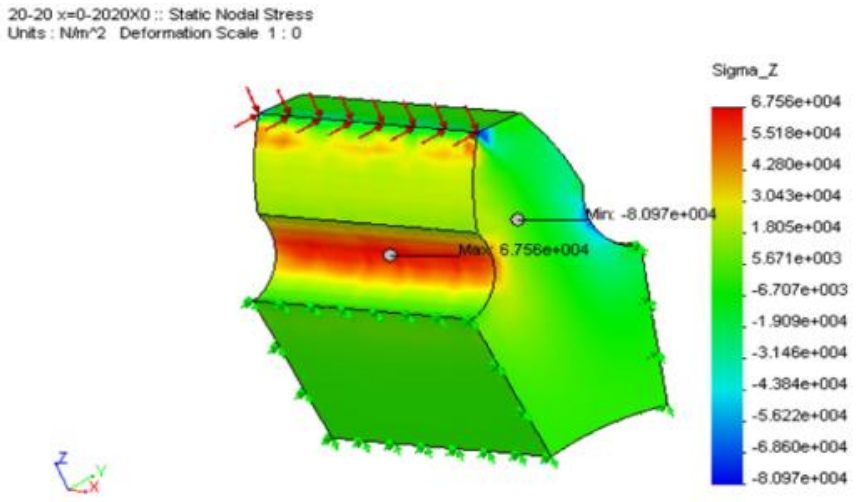

Fig. 5 Tensiones normales en el eje $Z\left(\sigma_{Z}\right)$ para ángulo del perfil del diente de 20 grados por ambas caras sin corrección.

En la Figura 6 se muestra el mismo diente, pero

ahora con un coeficiente de asimetría de 1,35.

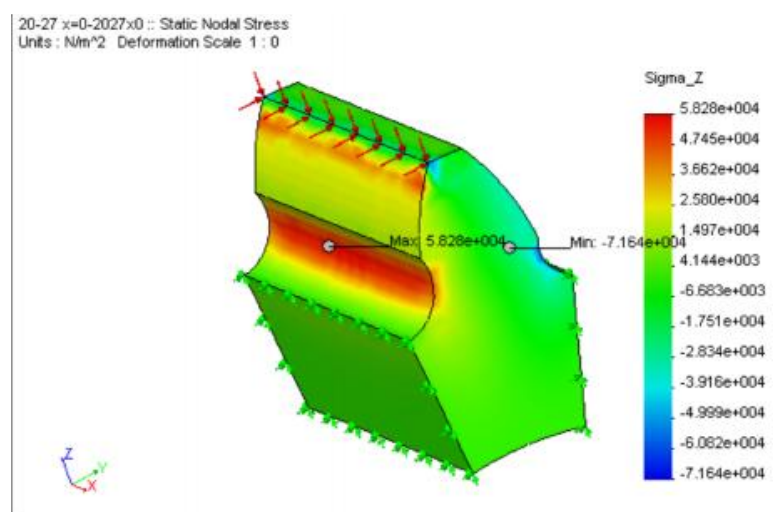

Fig. 6 Tensiones normales en el eje $Z\left(\sigma_{Z}\right)$ para ángulo de ataque del perfil del diente de 20 grados y de respaldo de 27 grados.

En las Figuras 7, 8 y 9 se muestran como varían coeficiente de asimetría y de la corrección para las tensiones normales en el eje $\mathrm{z}$ en función del diferentes ángulos del diente en la zona de ataque.

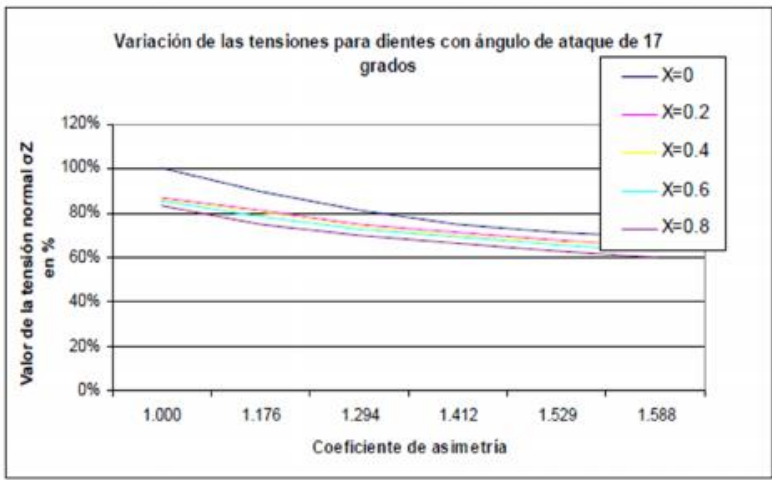

Fig. 7 Variación de las tensiones normales en el eje $Z(\sigma z)$ en función del coeficiente de asimetría y de la corrección para un ángulo del perfil del diente en la zona de ataque de 17 grados. 


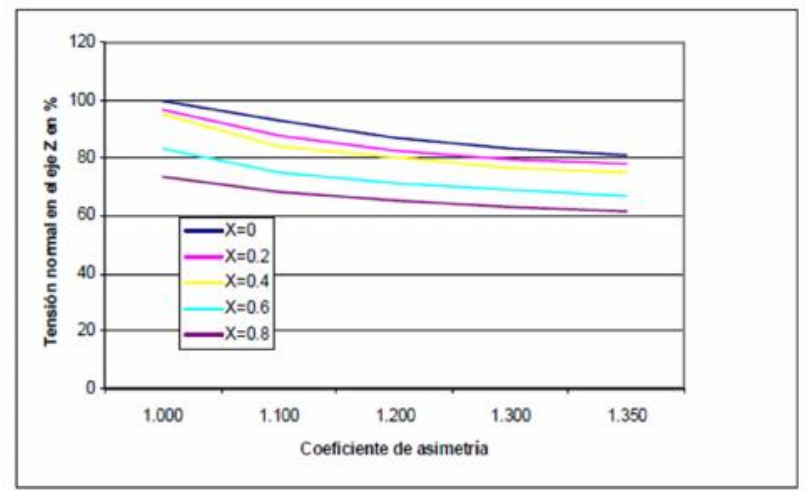

Fig. 8 Variación de las tensiones normales en el eje $Z(\sigma z)$ en función del coeficiente de asimetría y de la corrección para un ángulo del perfil del diente en la zona de ataque de $20^{\circ}$.

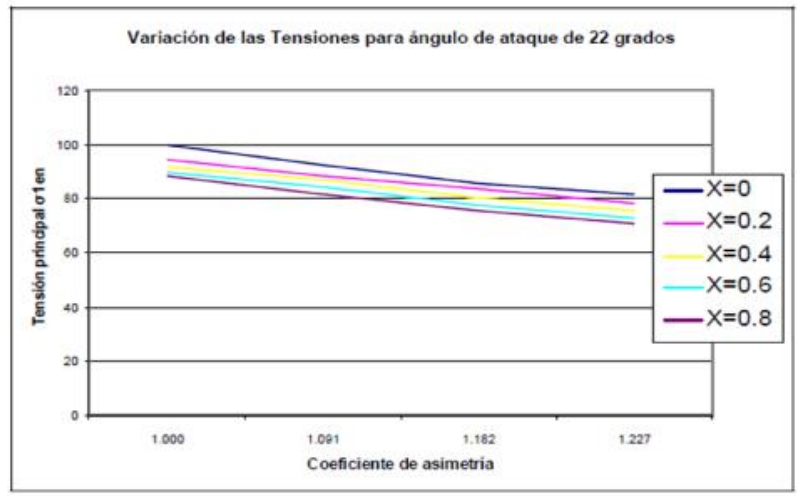

Fig. 9 Variación de las tensiones normales en el eje $Z\left(\sigma_{z}\right)$ en función del coeficiente de asimetría y de la corrección para un ángulo del perfil del diente en la zona de ataque de $22^{\circ}$.

Metodología de Cálculo de los engranajes plásticos propuesta por los autores.- Para hallar una expresión más exacta para el cálculo de tensiones en dientes asimétricos, se desarrolló un experimento virtual, utilizando el software Cosmos DesignStar. Para ello, se desarrollaron tres etapas:

Evaluación de la influencia del coeficiente de asimetría en la determinación de la tensión en el pie del diente.Se mantuvo el número de dientes fijo $(\mathrm{Z}=17)$, el factor de corrección igual a cero $(\chi=0)$, y se varió el coeficiente de asimetría desde 1 hasta 1,588. Se utilizó como material Nylon 6,10 y se utilizó una fuerza unitaria, un módulo unitario y una carga unitaria. Se determinaron entonces las tensiones máximas en el pie del diente. En la Tabla 3 se muestran los valores de Tensiones en el pie del diente para estas condiciones en función del coeficiente de asimetría:
Tabla 3. Variación de las tensiones en el pie del diente en función del coeficiente de asimetría.

\begin{tabular}{lc}
\hline $\mathrm{C}$ & $\sigma_{\mathrm{Z}(\mathrm{MPa})}$ \\
\hline 1 & 5.6 \\
1.05 & 5.35 \\
1.1 & 5.1 \\
1.15 & 4.84 \\
1.176 & 4.73 \\
1.2 & 4.63 \\
1.25 & 4.44 \\
1.3 & 4.27 \\
1.35 & 4.1 \\
1.412 & 3.98 \\
1.5 & 3.75 \\
\hline
\end{tabular}




3.629

Utilizando el Software Curve Expert 1,3 para Windows, se determinó la ecuación de la Tensión en función del coeficiente de asimetría:

$$
\sigma=a-b e^{-c \cdot C^{d}}
$$

Error Estándar: 0.014
Coeficiente de Correlación: 0.9998

Donde:

$\mathrm{a}=6.747125, \quad \mathrm{~b}=3.862122, \quad \mathrm{c}=1.219807, \quad \mathrm{~d}=-$ 3.85999, siendo $\mathrm{C}=$ Coeficiente de asimetría.

En la Figura 10, se muestra como varía esta función y el grado de aproximación de la misma se aprecia el buen ajuste de la curva obtenida.

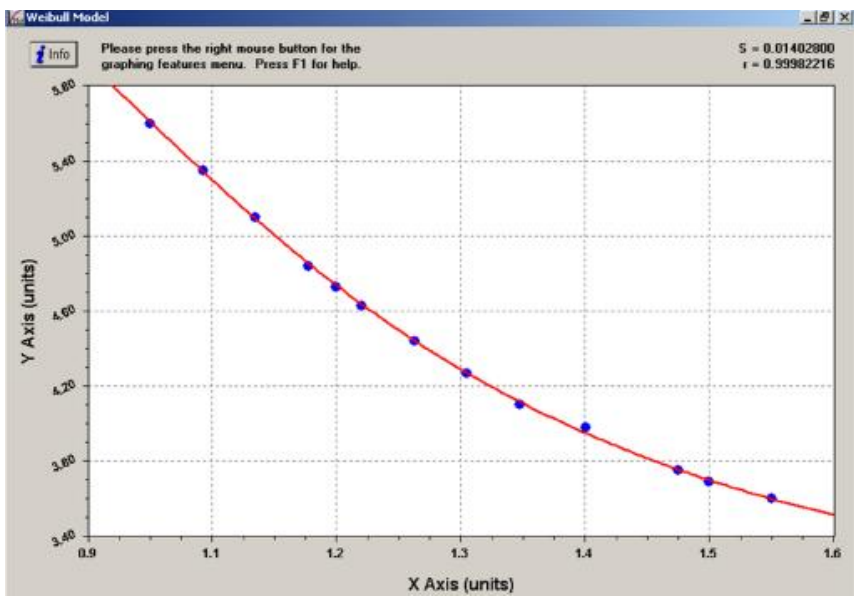

Tensión $(Y)$ vs. Coeficiente de asimetría $(X)$

Fig. 10 Tensión en función del coeficiente de asimetría.

Evaluación de la influencia del coeficiente de corrección en la determinación de la tensión en el pie del diente

Posteriormente se mantuvo el número de dientes fijo $(Z=17)$, el coeficiente de asimetría igual a 1 y se varió el coeficiente de corrección desde 0 hasta 0,7 . Se utilizó como material Nylon 6,10 y se utilizó una fuerza unitaria, un módulo unitario y una carga unitaria.

Se determinaron entonces las tensiones máximas en el pie del diente.

En la Tabla 4 se muestran los valores de Tensiones en el pie del diente para estas condiciones, en función del coeficiente de corrección.
Tabla 4. Variación de las tensiones en el pie del diente en función del coeficiente de corrección $(\chi)$. 
Utilizando el Software Curve Expert 1,3 para Windows, se determinó la ecuación de la Tensión en función del coeficiente de corrección:

$$
\sigma=a e^{b \chi}
$$

Error Estándar: 0.04304

Coeficiente de Correlación: 0.996304

Aquí: $\mathrm{a}=5.6219788$

$\mathrm{b}=-0.4456412$

$\chi=$ coeficiente de corrección

En la Figura 11 se muestra como varía esta función y el grado de aproximación de la misma, se aprecia el buen ajuste de la curva obtenida.

\begin{tabular}{|c|c|}
\hline & $\sigma_{\mathbf{z}}(\mathbf{M P a})$ \\
\hline 0 & 5.6 \\
\hline 0.05 & 5.50 \\
\hline 0.1 & 5.41 \\
\hline 0.15 & 5.30 \\
\hline 0.2 & 5.10 \\
\hline 0.25 & 4.95 \\
\hline 0.3 & 4.86 \\
\hline 0.35 & 4.81 \\
\hline 0.4 & 4.73 \\
\hline 0.45 & 4.68 \\
\hline 0.5 & 4.53 \\
\hline 0.55 & 4.38 \\
\hline 0.6 & 4.27 \\
\hline 0.65 & 4.20 \\
\hline 0.7 & 4.09 \\
\hline
\end{tabular}

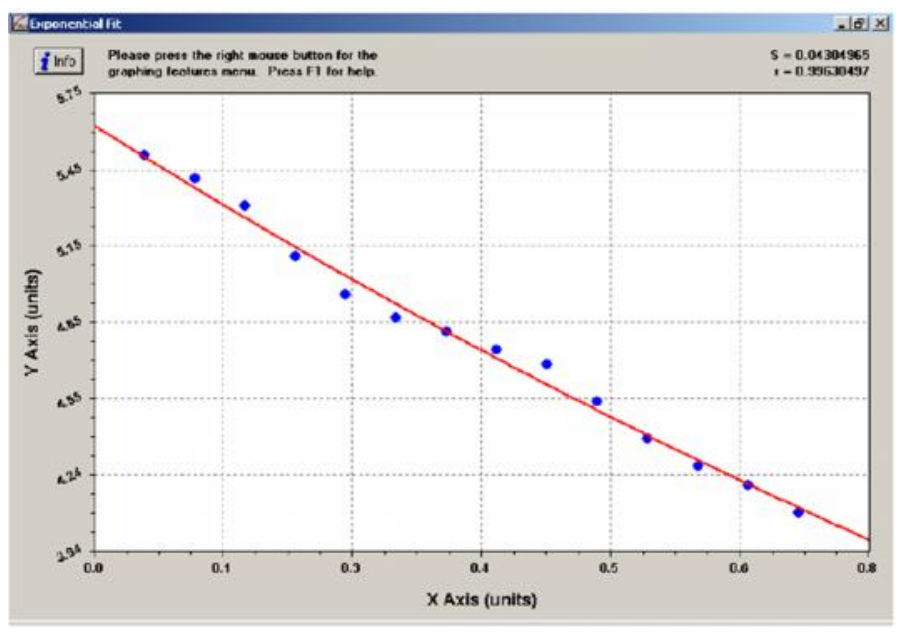

Tensión (Y) vs. Factor de corrección (X)

Fig. 11 Tensión en función del coeficiente ó factor de corrección.

Evaluación de la influencia del número de dientes en la determinación de la tensión en el pie del diente

Finalmente, se mantuvo el coeficiente de asimetría igual a 1, la corrección igual a cero, y se varió el número de dientes. Se utilizó como material Nylon 6,10 y se utilizó una fuerza unitaria, un módulo unitario y una carga unitaria. Se determinaron entonces las tensiones máximas en el pie del diente.

En la Tabla 5 se muestran los valores de Tensiones en el pie del diente para estas condiciones en función del número de dientes.

Tabla 5. Variación de las tensiones en el pie del diente en función del número de dientes.

\begin{tabular}{cc}
\hline $\mathrm{Z}$ & $\sigma_{\mathrm{z}}(\mathrm{MPa})$ \\
\hline 17 & 5.59 \\
21 & 5.52 \\
\hline
\end{tabular}




\begin{tabular}{rr}
\hline 29 & 5.12 \\
37 & 4.81 \\
43 & 4.40 \\
57 & 4.03 \\
67 & 3.85 \\
73 & 3.67 \\
83 & 3.63 \\
91 & 3.59 \\
\hline 1,3 para & Aquí $\mathrm{a}=1.3476586$ \\
la Tensión & $\mathrm{b}=-0.65875851$ \\
& $\mathrm{c}=0.0088964867$ \\
$\mathrm{Z}=$ número de dientes
\end{tabular}

$$
\sigma=\frac{a}{1+b e^{-c Z}}
$$

Error Estándar: 0.0532291

Coeficiente de Correlación: 0.9939859
En la Figura 12, se muestra como varía esta función y el grado de aproximación de la misma, de la misma se aprecia el buen ajuste de la curva obtenida.

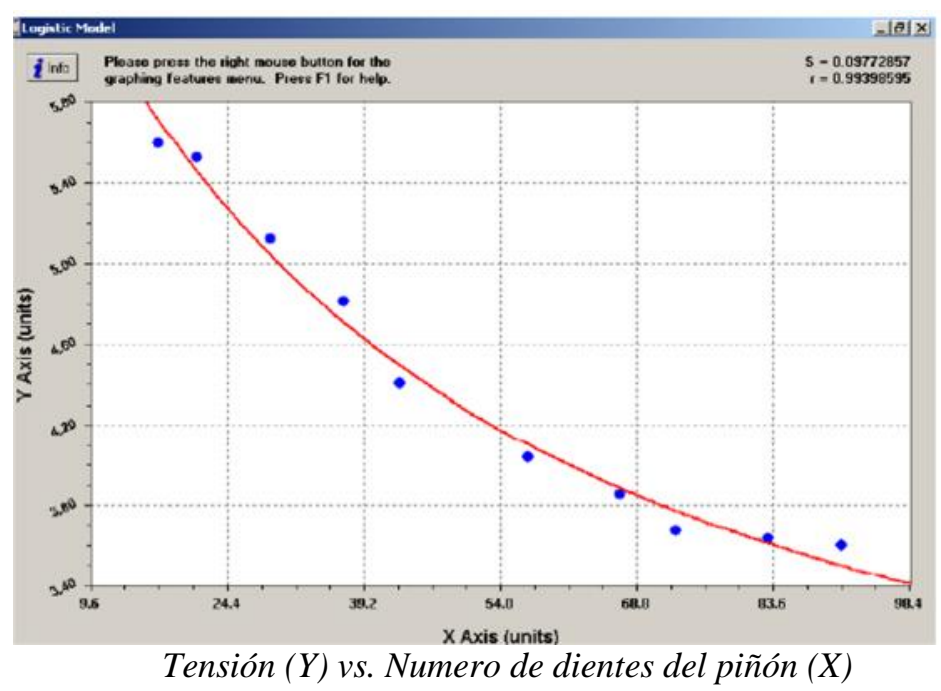

Fig. 12 Tensión en función del número de dientes del piñón.

Evaluación de la influencia simultanea del coeficiente de asimetría, factor de corrección y del número de dientes en la determinación de la tensión en el pie del diente.

Para evaluar la influencia simultánea de los tres parámetros $(\mathrm{X}, \mathrm{C}, \mathrm{Z})$, en el valor de las tensiones que surgen en el pie del diente, se utilizó el Software StatGraphics 4,1 Plus, realizándose un mayor número de experimentos.

Llegándose a la siguiente función (Para fuerzas, módulo y ancho unitarios):

$$
\sigma=1,33615-\frac{0,452994}{1-0,05 e^{0,0386 Z}}-3,12642 C^{\frac{0,662}{C}}+4,3 e^{0,142821} \cdots
$$


Entonces, el valor de la tensión real en un diente de un engranaje asimétrico será el valor calculado por la expresión anterior, afectada por la carga actuante, el módulo, el ancho de la rueda, y el factor de Lewis.
Llamando a la expresión anterior $\sigma_{\mathrm{r}}$, se obtiene:

$$
\sigma=\frac{F_{t}}{m \cdot b \cdot Y_{F}} C_{r}
$$

Luego:

$$
C_{r}=1,33615-\frac{0,452994}{1-0,05 e^{0,0386 Z}}-3,12642 C^{\frac{0,662}{C}}+4,3 e^{0,142821 \chi}
$$

Se programó todos los parámetros anteriores y para validar la expresión se desarrolló siete cálculos utilizando el software Cosmos DesignStar.
En la Tabla 6 se muestra los resultados de la misma en la que se puede apreciar el error de la expresión obtenida con respecto al método de los elementos finitos.

Tabla 6. Valores de las Tensiones calculadas por la expresión obtenida por el MEF.

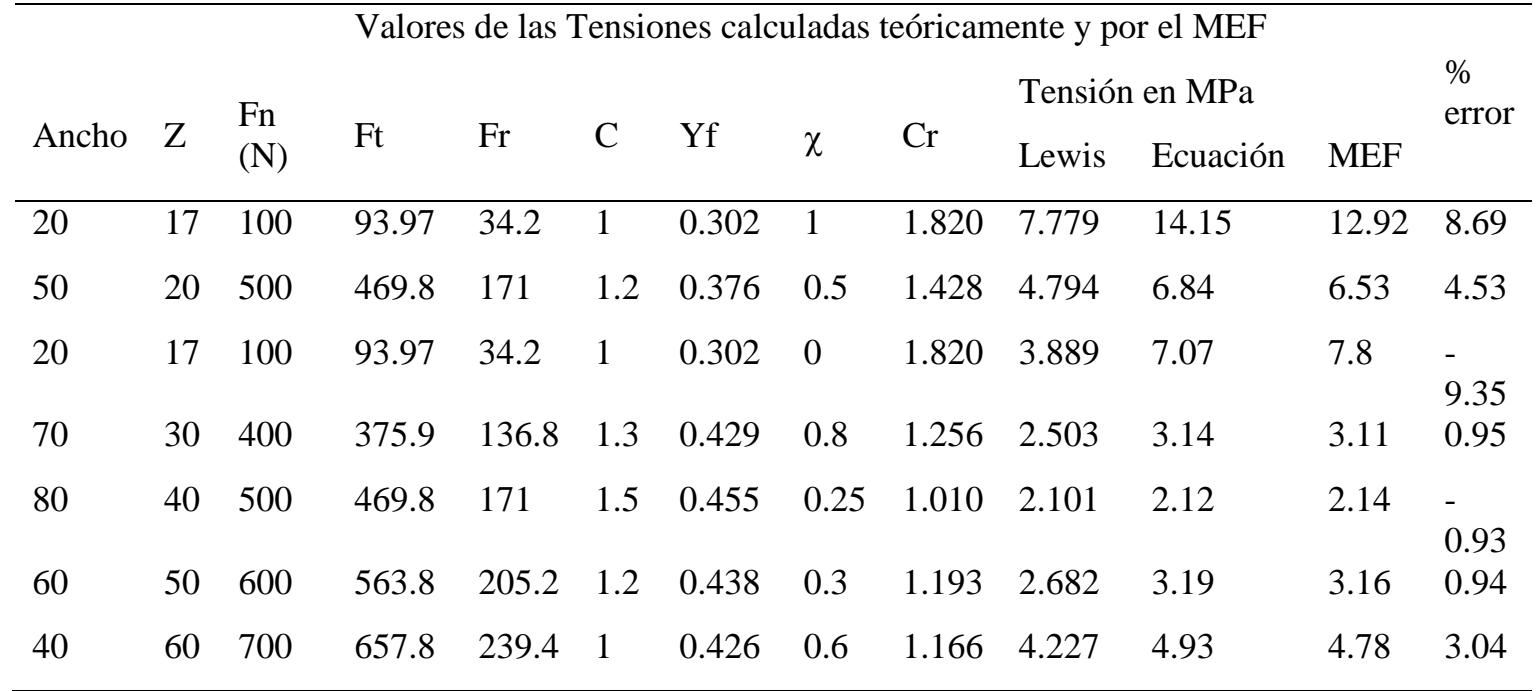

La expresión 7 fue transformada para usarla en el diseño de los engranajes cilíndricos de dientes rectos de materiales plásticos, adquiriendo la forma siguiente:

$$
m \geq \frac{C_{r} \cdot F_{t}}{[\sigma] \cdot b \cdot Y_{F}}
$$

Todos los términos de esta expresión ya han sido definidos anteriormente. Como en el momento de diseñar no se conoce el ancho de la rueda, se crea el ancho relativo con respecto al módulo $\left(\varphi_{\mathrm{m}}\right)$ cuyo valor varía de acuerdo a recomendaciones avaladas por la práctica entre 6 y 10 [12]. La expresión para determinar el módulo queda entonces como:

$$
m^{2} \geq \frac{C_{r} \cdot F_{t}}{[\sigma] \cdot \varphi_{m} \cdot b \cdot I_{F}}
$$

Teniendo en cuenta que la fuerza tangencial $F_{t}$ puede expresarse en función del momento torsor $\mathrm{M}_{\mathrm{t}} \mathrm{y}$ del diámetro de paso de la rueda se tiene: 


$$
F_{t}=\frac{2 M_{t}}{m \cdot Z_{p}}
$$

Finalmente la expresión para determinar el módulo quedará:

$$
m \geq \sqrt[3]{\frac{2 C_{r} \cdot M_{t}}{\varphi_{m} Y_{F}[\sigma] \cdot Z_{p}}}
$$

El valor del momento torsor está determinado previamente por las condiciones de potencia y velocidad angular como parámetros de entrada:

$$
\mathrm{M}_{\mathrm{t}}=9,55 \times 10^{6} \mathrm{~N} \cdot \mathrm{m}
$$

\section{RESULTADOS FINALES}

Resultados de la influencia de la asimetría en la resistencia a la flexión de los engranajes plásticos

En la actualidad para los engranajes plásticos, ninguno de los métodos de cálculo existentes tiene en cuenta la influencia de la asimetría y la corrección en la resistencia del diente a la flexión.
La norma AGMA para engranajes plásticos establece que la corrección tiene una influencia notable en la geometría y por tanto en la resistencia a la flexión de los engranajes plásticos. Sin embargo, no han precisado como disminuyen las tensiones principales al usar correcciones en los engranajes plásticos.

Autores tan renombrados en esta temática como Zan Smith [14] evadieron este aspecto. Sin embargo en el año 1998 Alexander Kapelevich publicó por primera vez la variante de usar engranajes asimétricos en materiales metálicos [6], iniciándose así una nueva etapa en las transmisiones por engranajes. No obstante aun todavía no se ha evaluado totalmente la influencia de la asimetría y de la corrección en la resistencia de los engranajes plásticos, razón de éste trabajo.

De los cálculos realizados en el apartado 5.0, podemos concluir acerca de la gran influencia de utilizar engranajes con dientes asimétricos en reemplazo de los simétricos dado que los esfuerzos se reducen en más del $18 \%$, según se muestra en la Tabla 7.Lo que constituye una mejora en la vida útil del engranaje, asimismo este trabajo realiza una innovación científica en este tema.

Tabla 7. Resumen de resultados comparativos del cálculo de los esfuerzos de flexión para engranajes simétricos y asimétricos sin corrección.

\begin{tabular}{lllllll}
\hline $\begin{array}{l}\text { Coef. de } \\
\text { asimetría }\end{array}$ & $\begin{array}{l}\text { Factor de } \\
\text { corrección }\end{array}$ & $\begin{array}{l}\text { Tipo } \\
\text { diente }\end{array}$ & de & $\begin{array}{l}\text { Ángulo } \\
\text { ataque }\end{array}$ & $\begin{array}{l}\text { Ángulo de } \\
\text { respaldo }\end{array}$ & $\begin{array}{l}\text { Tensión normal } \\
(\mathrm{Pa})\end{array}$ \\
\hline 1 & 0 & Simétrico & $20^{\circ}$ & $20^{\circ}$ & $6,756 \times 10^{4}$ \\
1,35 & 0 & Asimétrico & $20^{\circ}$ & $27^{\circ}$ & $5,526 \times 10^{4}$ \\
\hline
\end{tabular}

\section{Resultados de la influencia de la corrección del dentado en la resistencia a la flexión de los engranajes plásticos}

De los cálculos efectuados en la ecuación (4) se muestra los resultados de los esfuerzos sin y con corrección, según detalle en la Tabla 8.

Tabla 8. Resumen de resultados comparativos del cálculo de los esfuerzos de flexión para engranajes simétricos con corrección.

\begin{tabular}{ccc}
$\chi$ & $\sigma_{z}(\mathrm{MPa})$ & Reducción de esfuerzos $(\%)$ \\
\hline 0 & 5.6 & 0 \\
0.7 & 4.094 & 26,89 \\
\hline
\end{tabular}

$\mathrm{Al}$ haberse realizado las iteraciones para $\mathrm{Z}=17$ dientes, utilizando dientes simétricos, se puede constatar que el esfuerzo sin corrección en el pie del diente da como resultado $\sigma=5,6 \mathrm{MPa}$ y al hacer una corrección al diente con $\chi=0,7$ para la misma condición nos da como resultado un esfuerzo de $\sigma=4,094 \mathrm{MPa}$, lo que constituye una reducción de los esfuerzos en el pie del diente de 
un $26,89 \%$ lo que influye notablemente en una mejora en la vida útil del engranaje, constituyéndose este resultado en un beneficio para el sector industrial.

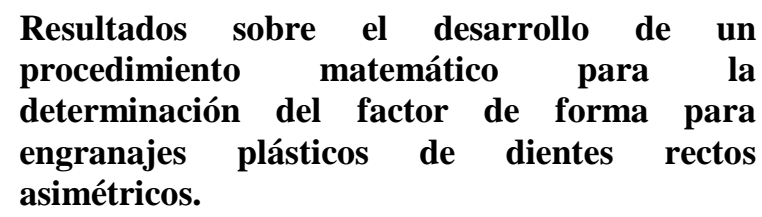

Se desarrolló un procedimiento matemático de la ecuación de Lewis en la expresión (1). De los cálculos efectuados con el algoritmo propuesto y en concordancia con los resultados de la Tabla A.1 se obtuvieron los valores del factor de forma o de Lewis (Y) para un mismo número de dientes, analizando la variación de dicho factor en función del coeficiente de asimetría.

Se muestran los resultados comparativos en la Tabla 9.

Tabla 9. Resumen de resultados comparativos del cálculo del factor de forma $(Y)$.

\begin{tabular}{ccc}
$\mathrm{Z}$ & $\mathrm{C}$ & $\mathrm{Y}$ \\
\hline & & \\
50 & 1,0 & 0,408 \\
& 1,2 & 0,438 \\
\hline
\end{tabular}

Según los resultados, podemos concluir que a un incremento del coeficiente de asimetría, aumenta el factor de forma o de Lewis, concluyéndose que disminuye significativamente el esfuerzo en el pie del diente en un $6,84 \%$, lo que implica la gran influencia de utilizar engranajes con dientes asimétricos en reemplazo de los simétricos, lo que constituye una mejora en la vida útil del engranaje.

\section{CONCLUSIONES}

De los resultados y discusiones se ha llegado a las siguientes conclusiones:

Se comprobó cómo la corrección produce dientes de mayor espesor en su base. Este incremento del espesor del diente alcanzo valores hasta del $26 \%$ mayor con respecto al espesor de un diente simétrico sin corrección, esto proporcionó un incremento de la capacidad de carga.

Se ha desarrollado una expresión matemática para determinar las tensiones que se producen en engranajes con dientes asimétricos, el cual, es muy diferente al factor de Lewis para dientes simétricos. Esta expresión desarrollada para la determinación de las tensiones en el pie del diente de los engranajes asimétricos ofrece resultados bastante cercanos a los obtenidos por el método de elementos finitos, lo que valida su aplicación en el sector industrial.

La asimetría tiene una gran influencia en la variación de las tensiones en el pie del diente, comprobándose que para un ángulo de ataque de $20^{\circ}$ y de respaldo $27^{\circ}$, se logró disminuciones de las tensiones en el pie del diente de un $18 \%$ con respecto al esfuerzo que corresponde a uno simétrico, proporcionando un incremento de la capacidad de carga.

\section{REFERENCIAS}

1. Mott, R. L., "Diseño de elementos de máquinas". Editorial Prentice Hall, 1995.

2. Deutschman, A. D., Michels, W, J., Wilson, Ch. E., "Diseño de máquinas". Editorial CECSA, 1985, pp. 973, México.

3. Fritzinger, D., "What to put on parts prints of plastic gears". Revista Machine Design. Vol. 70, Issue 20. Penton Publishing ISSN 00249114. 1998. pp. 114. USA.

4. Fuentes Aznar, A., "Modelo de Cálculo a Flexión de Engranajes Cilíndricos de Perfil de Evolvente". Tesis Doctoral, U.N.E.D. 1996. pp. 50-95, Madrid.

5. Garcia Masia, C., "Optimización del Diseño de Engranajes por Análisis Paramétrico". Tesis Doctoral. U.N.E.D. 1994. pp. 89-103. Madrid.

6. Gonzales Rey, G., "Apuntes para el Cálculo de Engranajes Cilíndricos según la norma I.S.O. del Comité Técnico 60. E.P.U. de Zamora. I.S.P.J.A.E”. 1988. pp. 55-67, Ciudad Habana.

7. Kapelevich, A., "Geometry and design of involute spur gears with asymmetric teeth". 1999 Elsevier Science Ltd. Pp. 117-130, URSS. 
8. Kleiss, R. E., Kapelevich A. L., Kleiss, Jr., "New Opportunities with Molded Gears". AGMA Fall Technical Meeting, Detroit, October 3-5, 2001, (01FTM9) Copyright _ 2001 American Gear Manufacturers Association 1500 King Street, Suite 201 Alexandria, Virginia, October, 2001 ISBN: 1-55589--788-6" - USA.

9. Kleiss, R., Scott Hoffman, J., "The Generation of Precision Spur Gears Through Wire Electrical Discharge Machining". Technical Conference of the AGMA. 1993. Minneapolis St. Paul, USA.

10. Kleiss, R. E., "Must You Use Metal?" Revista Design Engineering. Rogers Media, Publishing. One Mount Pleasant Road. Marzo 2001. pp: 7. Toronto, Ontario, Canada.

11. Lewis, W., "Investigation of Strenght of Gear teeth. Proc. of Engineers Club. P.A. 1982. pp. 16-23, Philadelphia.

12. Moya, J., Franco, R., Fernandez, F. "Particularidades del cálculo de engranajes plásticos". UCLV.
13. Moya, J., Franco, R., "Diseño de engranajes con materiales plásticos, una vía de preservar el Medio Ambiente". Memorias de la II Conferencia "Medio Ambiente Siglo XXI". Noviembre del 2001 Santa Clara, Villa Clara, Cuba.

14. Smith, Z., "Gearing up with plastics. Revista Mechanical Engineering". Summit, ASME. 1998, New Jersey, USA.

15. Thomas, Y., CH, D., Yang, Shih-His Tang, "Design of new tooth profile for high load capacity gears". Mechanism and Machine Theory". Vol. 36, Issue 10. October 2001. pp: 1105-1120, Los Angeles, USA.

16. Yoerkie Charles A., Chory A. G., "Acoustic vibration characteristics of high contact ratio planetary gears". The Journal of American Helicopter Society. 40 (1984). pp. 19-32.

Correspondencia: titovilchez@uni.edu.pe

Recepción de originales: noviembre 2012 Aceptación de originales: marzo 2013 